\title{
CrystEngComm
}

Check for updates

Cite this: CrystEngComm, 2018, 20, 2449

Received 16th January 2018,

Accepted 20th March 2018

DOI: $10.1039 / \mathrm{c} 8 \mathrm{ce} 00074 \mathrm{c}$

rsc.li/crystengcomm

\section{Single crystal growth, transport and scanning tunneling microscopy and spectroscopy of $\mathrm{FeSe}_{1-x} \mathrm{~S}_{x} \dagger$}

\author{
Dmitriy Chareev, ${ }^{\text {abc }}$ Yevgeniy Ovchenkov, iD d Larisa Shvanskaya, iD de

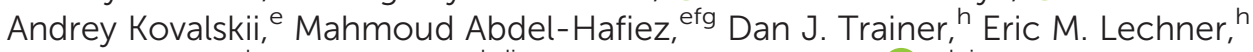 \\ Maria lavarone, ${ }^{\text {h }}$ Olga Volkova ${ }^{\text {bdi }}$ and Alexander Vasiliev iD *dei
}

\begin{abstract}
Single crystals of sulfur-substituted iron selenide, $\mathrm{FeSe}_{1-x} \mathrm{~S}_{x}$, were grown within eutectics of molten halides, $\mathrm{AlCl}_{3} / \mathrm{KCl}, \mathrm{AlCl}_{3} / \mathrm{KCl} / \mathrm{NaCl}$ or $\mathrm{AlCl}_{3} / \mathrm{KBr}$, under permanent temperature gradient. The innovative "ampoule in ampoule" design of a crystallization vessel allows obtaining $\mathrm{mm}$-sized plate-like single crystals with a sulfur content up to $x \sim 0.19$. The sharp anomalies in the physical properties indicate the superconducting and nematic phase transitions in $\mathrm{FeSe}_{0.96}$ at $T_{\mathrm{C}}=8.4 \mathrm{~K}$ and $T_{\mathrm{N}}=90 \mathrm{~K}$, respectively. Scanning tunneling microscopy reveals the presence of dumbbell defects associated with Fe vacancies and dark defects at the chalcogen site associated with $\mathrm{S}$ within the $\mathrm{FeSe}_{1-x} \mathrm{~S}_{x}$ series of compounds. Scanning tunneling spectroscopy shows the presence of two different superconducting gaps at both hole and electron pockets of the Fermi surface for low $S$ content levels. As a function of sulfur content, $T_{C}$ follows the conventional dome-shaped curve while $T_{N}$ decreases with $x$. The overall appearance of the $T-x$ phase diagram of $\mathrm{FeSe}_{1-x} \mathrm{~S}_{x}$ suggests the importance of nematic fluctuations for the formation of the superconducting state in these compounds.
\end{abstract}

\section{Introduction}

Iron selenide, $\mathrm{FeSe}_{1-\delta}$, exhibits intriguing and distinctive properties, which are currently in the research focus in the field of high temperature superconductors. This binary compound undergoes tetragonal-to-orthorhombic transition at about $90 \mathrm{~K}$ (ref. 1) and possesses an electronic nematic order below this temperature similar to many other Fe-based parent materials. However, no magnetic order is formed at ambient pressure $^{2}$ and it becomes superconducting at about 8-9 K. ${ }^{3}$ It shows superconductivity in a nearly stoichiometric form and remains a Pauli paramagnet down to the lowest temperatures. The superconducting transition temperature of this material can be increased (to $15-27 \mathrm{~K}$ ) by pressure ${ }^{4}$ and reaches over

\footnotetext{
${ }^{a}$ RAS, Institute of Experimental Mineralogy, Chernogolovka 123456, Russia

${ }^{b}$ Ural Federal University, Ekaterinburg 620002, Russia

${ }^{c}$ Kazan Federal University, Kazan 420008, Russia

${ }^{d}$ Lomonosov Moscow State University, Moscow 119991, Russia.

E-mail: vasil@mig.phys.msu.ru; Fax: +7 49593292 17; Tel: +7 4959329217

${ }^{e}$ National University of Science and Technology "MISiS", Moscow 119049, Russia

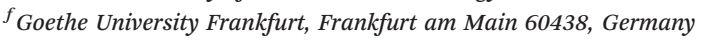

${ }^{g}$ Fayoum University, Fayoum 63514, Egypt

${ }^{h}$ Temple University, Philadelphia, PA 19122, USA

${ }^{i}$ National Research South Ural State University, Chelyabinsk 454080, Russia

$\dagger$ Electronic supplementary information (ESI) available. See DOI: 10.1039/ c8ce00074c
}

$45 \mathrm{~K}$ in a nanoscale FET type device. ${ }^{5}$ This places iron selenide on the verge of high temperature superconductivity and stimulates interest to study related and substituted series of compounds. Among these, the isovalent substitution of Se by $\mathrm{S}$ is supposed to produce a mild distortion in the crystal structure being, therefore, of considerable interest. The synthesis of $\mathrm{FeSe}_{1-x} \mathrm{~S}_{x}$ single crystals up to the highest possible concentration of sulfur is considered to be an important part of the comprehensive research of Fe-based superconductors as this system allows investigation of the relationship between superconductivity and nematicity. Indeed, nematicity can be systematically suppressed by the isovalent $S$ substitution. Moreover, sulfur substitution to FeSe enhances the upper critical field $H_{\mathrm{C} 2}$ and the critical current density. $T_{\mathrm{C}}$ initially increases slightly to $T_{\mathrm{C}} \approx 11 \mathrm{~K}$ at $\mathrm{S} \sim 10 \%$ from $T_{\mathrm{C}} \approx$ $8.5 \mathrm{~K}$ at $x=0$ but then decreases. ${ }^{6}$

There are several experiments that have explored the electronic properties of $\mathrm{FeSe}_{1-x} \mathrm{~S}_{x}$. Angle-resolved photoemission spectroscopy (ARPES) measurements ${ }^{7,8}$ have revealed that the size of the Fermi surface increases and that the anisotropy decreases by increasing $x$. An anisotropic superconducting gap was reported by ARPES in a $4 \%$ substituted sample $^{9}$ but, in general, the $x$ ranges studied so far are very limited.

According to the phase diagram of the Fe-Se system, superconducting tetragonal $\mathrm{FeSe}_{1-\delta}$ co-exists with metallic 
iron and the $\mathrm{h}-\mathrm{Fe}_{1-\Delta}$ Se hexagonal phase. ${ }^{10}$ At $457{ }^{\circ} \mathrm{C}$, the tetragonal phase is decomposed by the peritectoid reaction $\mathrm{FeSe}_{1-\delta}=\mathrm{h}-\mathrm{Fe}_{1-\Delta} \mathrm{Se}+\mathrm{Fe}$. The homogeneity region of $\mathrm{FeSe}_{1-x}$ is very narrow, and the range of possible compositions is from $\mathrm{FeSe}_{0.96}$ to $\mathrm{FeSe}_{0.975}{ }^{11} \mathrm{FeS}_{1-\delta}$, isostructural to FeTe and FeSe $(P 4 / \mathrm{nmm} ; a=3.674 \AA, c=5.033 \AA)$, is a mackinawite mineral. ${ }^{12}$ The exact composition of both natural and synthetic mackinawite is unknown and its thermodynamic stability has not been proven. Natural mackinawite decomposes forming pyrrhotite at $120-135{ }^{\circ} \mathrm{C} .{ }^{13,14}$ The superconductivity of synthetic mackinawite was described in ref. 15. The low stability of mackinawite makes the synthesis of stable $\mathrm{Fe}\left(\mathrm{Se}_{1-x} \mathrm{~S}_{x}\right)_{1-\delta}$ solid solutions difficult, especially at large $x$.

Although phase relations within the mixture of FeSe and FeS are not studied in detail, it is known that tetragonal iron selenide with any sulfur content is in equilibrium with metallic iron at any temperature. Taking into account that FeSe decomposes at $457{ }^{\circ} \mathrm{C}$ and tetragonal FeS is possibly not stable, one can explain the absence of complete sulfur substitution of selenium in FeSe. Thus, the temperature for the synthesis of iron selenide should not exceed $457^{\circ} \mathrm{C}$, and the maximum temperature for the synthesis of tetragonal $\mathrm{Fe}(\mathrm{Se}, \mathrm{S})_{1-\delta}$ solid solution should decrease with the increase of sulfur content.

\section{Experimental section}

The technique chosen for the growth of $\mathrm{Fe}(\mathrm{Se}, \mathrm{S})_{1-\delta}$ crystals was synthesis in eutectic molten metal chlorides under permanent temperature gradient. ${ }^{16,17}$ Molten chlorides constitute a very low melting eutectic mixture $\mathrm{AlCl}_{3} / \mathrm{KCl}\left(\mathrm{K}_{1} \mathrm{Al}_{2} \mathrm{Cl}_{7}\right)$, $\mathrm{AlCl}_{3} / \mathrm{KCl} / \mathrm{NaCl}\left(\mathrm{Na}_{0.5} \mathrm{~K}_{0.5} \mathrm{Al}_{2} \mathrm{Cl}_{7}\right)$ or $\mathrm{AlCl}_{3} / \mathrm{KBr}\left(\mathrm{K}_{1} \mathrm{Al}_{2} \mathrm{Cl}_{6} \mathrm{Br}_{1}\right)$. The permanent gradient is formed when the quartz ampoule with the chalcogen feed and the mixture of haloid salts is placed into a furnace and the temperatures at the ends of the ampoule are different due to the ampoule position. The feed gradually dissolves in the hot end of the ampoule and deposits in the cold end in the form of single crystals. The major advantage of this technique is that the physical and chemical parameters of crystal growth are strictly specified and constant in time unlike the classical solution-melt technique, when the multicomponent system gradually cools producing the single crystals. ${ }^{18}$ Previously, this method has been used to obtain high quality crystals of superconducting iron selenide $\mathrm{FeSe}_{1-\delta} \cdot{ }^{19}$

To grow the single crystals of sulfur-substituted iron selenide, the feed of $\mathrm{Fe}_{1.3} \mathrm{Se}_{1-x} \mathrm{~S}_{x}$ composition, $x=0.05-0.40$, was synthesized. A stable excess of iron increases the chemical potential of iron in the system preventing the formation of hexagonal h-Fe ${ }_{1-\Delta}(\mathrm{Se}, \mathrm{S})$.

Sometimes, pieces of metallic iron were added to the cold end of the ampoule to increase its chemical potential. This also leads to conditions suitable for the formation of tetragonal rather than hexagonal iron chalcogenides. To avoid possible mutual influence of sulfur and selenium ions during migration from the hot end of the ampoule to the cold end, the "ampoule in ampoule" design was employed, as shown in

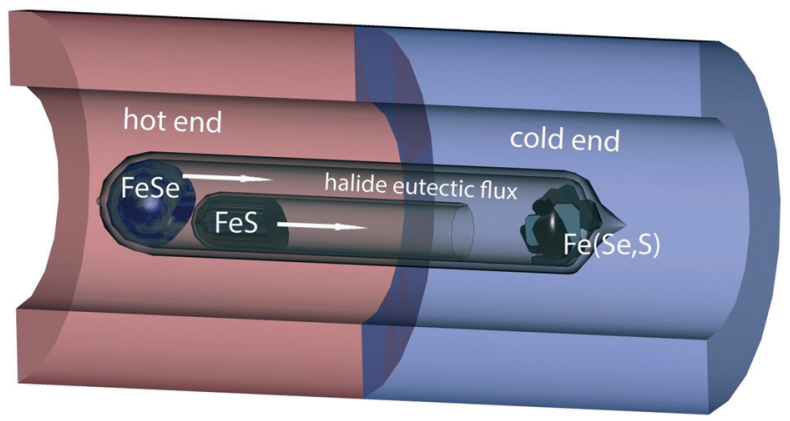

Fig. 1 "Ampoule in ampoule" design for the crystal growth of $\mathrm{FeSe}_{1-x} \mathrm{~S}_{x}$. The temperature decreases from the left (hot) end to the right (cold) end.

Fig. 1. This approach was introduced earlier to grow coexisting single crystals of FeAsS $+\mathrm{FeS}_{2}$, where As and S migration routes were separated, and (Fe,Ni)Te crystals with Fe and Ni separation. ${ }^{20}$

The feed of bulk composition of $\mathrm{Fe}_{1.3} \mathrm{Se}$ was placed into a thick-walled ampoule of $11 \mathrm{~mm}$ in inner diameter while the feed of $\mathrm{Fe}_{1.3} \mathrm{~S}$ was placed into an open thin-walled ampoule of $8 \mathrm{~mm}$ in outer diameter. Then, anhydrous $\mathrm{AlCl}_{3}$ was added to single or double ampoules, followed by air-stable alkali halides, e.g. $\mathrm{KBr}$ or a $\mathrm{KCl}-\mathrm{NaCl}$ mixture. Small amounts of these salts slightly decrease the melting point of aluminum chloride and prevent melting and evaporation of aluminum chloride during evacuation and sealing of the ampoules. The ampoules were placed into tube furnaces so that the end with no feed was at the edge of the furnace in order to create a temperature gradient of about $50-100{ }^{\circ} \mathrm{C}$. The furnaces were slowly heated to 400-420 ${ }^{\circ} \mathrm{C}$ and then were left under these conditions for 78 weeks. Since the solubility range of sulfur in iron selenide strongly depends on the crystallization temperature, the ampoules longer than $150 \mathrm{~mm}$ were used sometimes.

Double ampoules often exploded during heating, sometimes with detonation of the other ampoules. To prevent this, ampoules were heated in two stages: on the first day up to $250{ }^{\circ} \mathrm{C}$ and on the second day up to the final temperature of synthesis. During the night, the melted aluminum chloride had sufficient time to dissolve other salts which decreased its vapor pressure. Moreover, it was noticed that the explosion may be prevented by using the feed in the form of compressed pellets of corresponding size instead of powder. Synthesized crystals were washed from molten salts in water, then in ethanol and afterwards in acetone using an ultrasonic bath and then were dried for a short time in dry boxes at $70{ }^{\circ} \mathrm{C}$. As shown in Fig. 2, flat tetragonal crystals of about $\mathrm{mm}$ in size with thickness of about tenths of $\mu \mathrm{m}$ were obtained in all single ampoules when the sulfur content in the feed did not exceed $20 \%$. In the ampoules with higher sulfur content, hexagonal magnetic crystals were obtained routinely or no transport of the substance was observed. The crystals were stored in a vacuum.

In double ampoules, tetragonal crystals (Fig. 2a) or less often hexagonal crystals (see Fig. S1 of the ESI $\dagger$ ) were also 


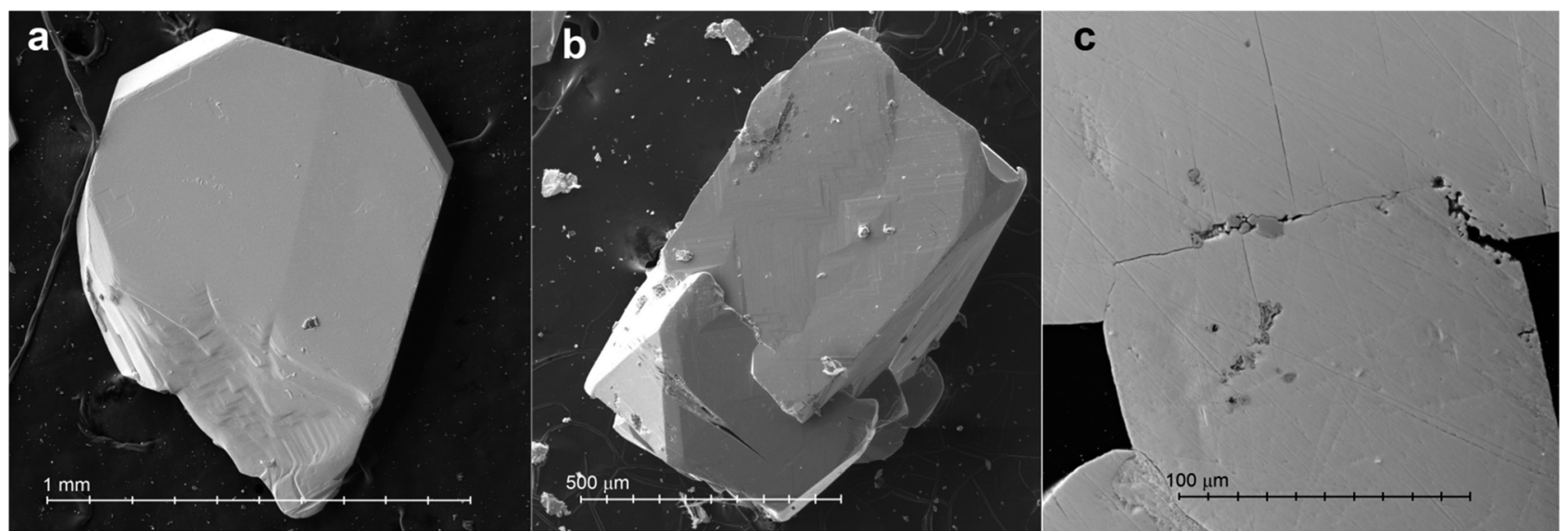

Fig. 2 Secondary electron images of a $\mathrm{FeSe}_{0.96} \mathrm{~S}_{0.04}$ single crystal. (a) $\mathrm{FeSe}_{0.96} \mathrm{~S}_{0.04}$ single crystal; (b) tridimensional $\mathrm{Fe}(\mathrm{Se}, \mathrm{S}$ ) crystal; (c) Fe $\mathrm{Si} \mathrm{Si}$ (darkgray) on the boundaries of $\mathrm{Fe}(\mathrm{Se}, \mathrm{S})$ crystals (light-gray).

found. In each ampoule, the tetragonal crystals were either flat and indistinguishable in shape, compared to those grown in single ampoules, or tridimensional, which often formed co-oriented sintered crystals (Fig. 2b). The difference in crystal shapes can be explained by the different degrees of supersaturation of the molten salt due to the interaction of dissolved ions of selenium and sulfur. In sintered $\mathrm{Fe}(\mathrm{Se}, \mathrm{S})$ crystals, particles of the approximate composition $\mathrm{Fe}_{2} \mathrm{Si}$ up to $10 \mu \mathrm{m}$ in size (Fig. 2c) were often found. Usually the sulfur content was from 2 to $19 \%$, but in one experiment a millimeter-size crystal containing $22 \pm 1 \%$ sulfur was found among small crystals. Microprobe analysis has shown that tetragonal crystals had an insignificant excess of iron compared to the combined content of sulfur and selenium. No impurity elements ( $\mathrm{Al}, \mathrm{K}, \mathrm{Na}, \mathrm{Cl}, \mathrm{Br}$ ) were found. The sulfur content in tetragonal crystals was always approximately two times lower than that in the feed. The use of long ampoules did not help to increase the sulfur content in the samples but increased the total amount of the transported substance.

The dependence of the ratio of iron to the sum of selenium and sulfur on the sulfur content $(\mathrm{Fe} /(\mathrm{Se}+\mathrm{S}) v s$. S) was studied for the crystals of five different compositions by microprobe analysis. It was observed that the iron-tochalcogen ratio remains constant within the error limits. It was the same as in pure tetragonal iron selenide and was equal to $0.92 \pm 0.04$. The difference in the non-stoichiometry obtained in this study and studies dealing with powder samples $(\sim 0.96)$ can be explained by the systematic error of X-ray fluorescence microanalysis or by oxidation of the surface of the polished crystal.

The lattice constants were determined using an Xcalibur-S area detector diffractometer with Mo $\mathrm{K}_{\alpha}$ radiation. According to the single-crystal X-ray characterization, all the samples belong to the tetragonal phase with lattice constants close to those reported for the polycrystalline samples of $\mathrm{FeSe}_{1-\delta \cdot}{ }^{21}$ Fig. 3 presents the variation of lattice constants $a$ and $c$ in the $\mathrm{FeSe}_{1-x} \mathrm{~S}_{x}$ samples. The XRD pattern of $\mathrm{FeSe}_{0.9} \mathrm{~S}_{0.1}$ is shown in Fig. S2 of the ESI.† Our data show that the substitu- tion of Se by $S$ results in the decrease of both in-plane lattice parameter $c$ and inter-plane lattice parameter $a$. The crystal lattice parameters within the $\mathrm{FeSe}_{1-x} \mathrm{~S}_{x}$ series of compounds are collected in Table $\mathrm{S} 1$ of the ESI. $\dagger$ Similar results have been reported for powder $\mathrm{FeSe}_{1-x} \mathrm{~S}_{x}(0.1<x<0.5)$ samples. $^{22}$

\section{Scanning tunneling microscopy and spectroscopy}

Low temperature scanning tunneling microscopy (STM) and spectroscopy (STS) measurements have been performed using a Unisoku UHV STM system, with a base pressure of $4 \times$ $10^{-11}$ Torr. The crystals were cleaved under UHV at room temperature and soon after were transferred to the STM scanner at low temperature. Pt-Ir tips were used in all experiments.

Atomically resolved images were acquired in the constant current mode. The STM topography image acquired at the surface of a pure FeSe single crystal at $T=1.5 \mathrm{~K}$ is shown in the left panel of Fig. 4. The image reveals the presence of dimer-like defects (bright spots) surrounded by dark regions that are usually associated with Fe vacancies. ${ }^{23}$ Similar dumbbell defects have also been imaged in other FeSe crystals grown by vapor transport. ${ }^{24,25}$

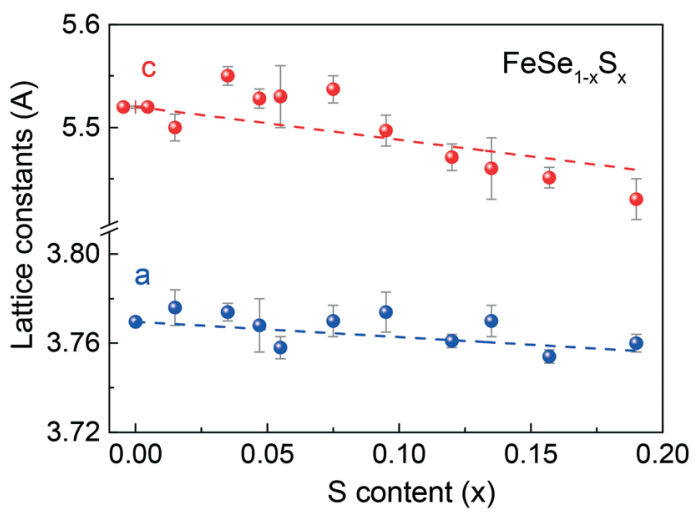

Fig. 3 The lattice constants $a$ and $c$ of the $\mathrm{Fe}\left(\mathrm{Se}_{1-x} \mathrm{~S}_{x}\right)_{1-\delta}$ tetragonal single crystals as a function of $\mathrm{S}$ content. The data values for parent compound $\mathrm{FeSe}_{1-\delta}$ are taken from ref. 15. 

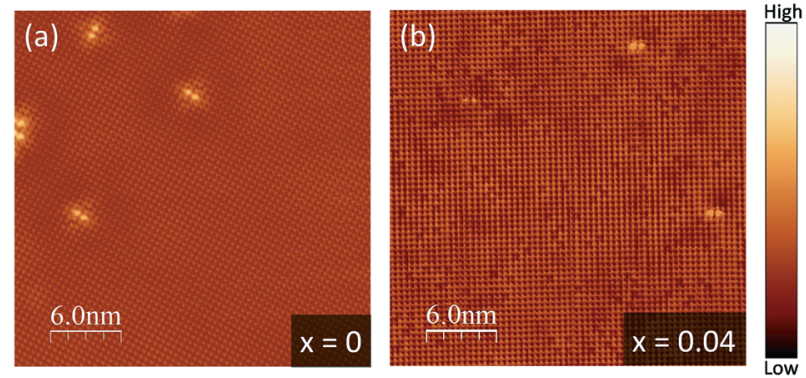

Fig. 4 (a) Constant-current STM image of a cleaved (001) FeSe single crystal. Scanning parameters are $V=25 \mathrm{mV}$ and $I=60 \mathrm{pA}$. The scanning direction is at approximately 45 degrees with respect to the topmost Se atoms. (b) STM image of $\mathrm{FeS}_{0.04} \mathrm{Se}_{0.96}$. Scanning parameters are $V=-20 \mathrm{mV}$ and $I=100 \mathrm{pA}$. The scanning direction is almost parallel to the topmost Se lattice. In both images, the scan area is $23.5 \mathrm{~nm} \times$ $23.5 \mathrm{~nm}$.

They are aligned both along the $a$ - and $b$-axes, which point to their independence from a structural orthorhombic distortion. ${ }^{26}$ The STM topography image acquired at the surface of a $\mathrm{FeSe}_{0.96} \mathrm{~S}_{0.04}$ single crystal is shown in the right panel of Fig. 4. Also in this case dumbbell defects are present with a similar concentration. Dark defects at the chalcogen site are associated with the $\mathrm{S}$ atoms that have smaller radii than the Se atoms. The density of these defects corresponds closely to the nominal concentration of sulfur in the measured sample. These kinds of defects are absent in the pure FeSe sample.

Conductance spectra, $\mathrm{d} I / \mathrm{d} V$, which are proportional to the local electronic density of states, have been acquired using the lock-in $a c$ modulation technique while the $I-V$ curves were recorded simultaneously. A typical tunneling spectrum acquired on the surface of FeSe at $T=1.5 \mathrm{~K}$ is shown in the left panel of Fig. 5. The spectrum presents clear coherence peaks at $\Delta_{1}= \pm 2.5 \mathrm{meV}$ that have been observed in other STM measurements both on thin films ${ }^{27,28}$ and on single crystals. $^{24,25,29}$ This gap is usually associated with the gap present on the $\Gamma$-pocket, as the tunneling probability is much higher for tunneling into the $\Gamma$ hole-pocket. Another feature is also present in the spectrum as a kink at a lower energy $\left(\Delta_{2}= \pm 1.1\right.$ $\mathrm{meV}$ ), and we associate this with the gap of the electron-

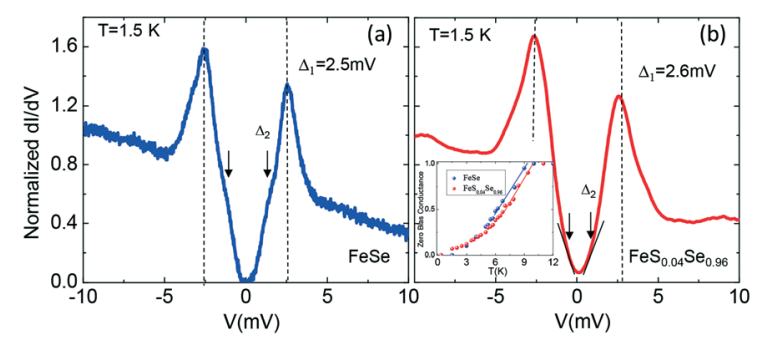

Fig. 5 Tunneling spectra $\mathrm{d} / / \mathrm{d} V$ acquired at $T=1.5 \mathrm{~K}$ (a) on FeSe and (b) $\mathrm{FeS}_{0.04} \mathrm{Se}_{0.96}$. Tunneling conditions are $V=10 \mathrm{mV}, I=60 \mathrm{pA}$, lockin modulation $V_{\text {mod }}=0.2 \mathrm{mV}$ and lock-in frequency $f=373.1 \mathrm{~Hz}$. The tunneling spectra have been normalized to $V=-10 \mathrm{mV}$. The inset in (b) is the evolution of zero bias conductance as a function of temperature for the two samples and shows a shift of the critical temperature of the $\mathrm{FeS}_{0.04} \mathrm{Se}_{0.96}$ sample to higher temperatures. pocket. A typical spectrum acquired on a $\mathrm{FeSe}_{0.96} \mathrm{~S}_{0.04}$ single crystal at $T=1.5 \mathrm{~K}$ is shown in the right panel of Fig. 5 . The spectrum presents coherence peaks corresponding to the $\Gamma$ hole-pocket at a slightly higher energy $\left(\Delta_{1}=2.6 \mathrm{meV}\right)$ and the lower energy feature at a slightly lower energy $\left(\Delta_{2}= \pm 0.9\right.$ $\mathrm{meV})$.

In order to extract the superconducting transition temperatures, we plotted the zero bias conductance (ZBC) as a function of temperature in the inset to the right panel of Fig. 5 for both samples. The ZBC of the tunneling spectra is the electronic density of states at the Fermi level. As the temperature increases and the gap closes, the $\mathrm{ZBC}$ value increases to 1 in the normal state. Taking the intersection point of two straight lines, fitting the $\mathrm{ZBC}$ values in the range of 6-9 $\mathrm{K}$ and the higher temperature region, we can estimate the transition temperature $T_{\mathrm{C}}$. We find that the transition temperature increases from the pure sample to the $4 \% \mathrm{~S}$ substituted sample by about $1 \mathrm{~K}$. While the transition temperatures determined with this method might not be precise, they show a clear trend which is in agreement with the transport data.

At low S substitution (4\%), we find that the spectroscopic features are associated with the $\Gamma$-hole gap and those of the electron pocket are almost unchanged within the thermal broadening of the tunneling spectrum. These results are in agreement with previous findings reported with similar substitution levels by $\mathrm{STM}^{30}$ and ARPES. ${ }^{9}$ This suggests that at low S substitution levels, the strength of the superconducting pairing is not significantly affected by the initial suppression of the nematic order and the reduced anisotropy of the Fermi surface reported by ARPES. ${ }^{7}$

\section{Phase diagram}

The overall composition-temperature phase diagram is shown in Fig. 6. The temperature of the superconducting phase transition $T_{\mathrm{C}}$ was determined in the measurements of magnetization, specific heat and resistivity. ${ }^{6,31,32}$ In the range $x=0-0.19$, the composition dependence of the superconducting transition temperature exhibits a conventional

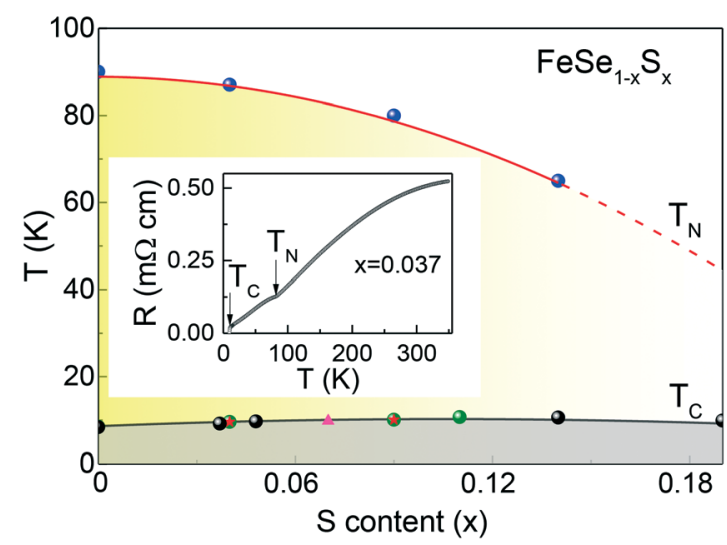

Fig. 6 Temperature-composition phase diagram for the $\mathrm{FeSe}_{1-x} \mathrm{~S}_{x}$ series of compounds. 
dome shape. The temperature of the nematic phase transition, determined by the position of a kink on $R(T)$, decreases with sulfur content which is in agreement with ref. 28. For $x$ $=0.037$, the temperature dependence of resistivity is shown in the inset to Fig. 6 . At $x=0.19$, the singularity on $R(T)$ was not detected reflecting the full suppression of the nematic phase transition.

No long-range magnetic order can be observed at ambient pressure in $\mathrm{FeSe}_{1-x} \mathrm{~S}_{x}$, which implies an isolated nematic quantum critical point. ${ }^{33}$ It has been reported that the spin density wave order can also be induced in $\mathrm{FeSe}_{1-x} \mathrm{~S}_{x}$ when physical pressure is applied. ${ }^{34}$ However, at higher $x$, the spin density wave phase can be completely decoupled from the nematic phase under pressure. Therefore, the chemical pressure introduced by $\mathrm{S}$ substitution offers an important route to separate the effects of nematic and magnetic fluctuations, allowing a discussion of their relative influence on superconducting pairing.

\section{Conclusions}

FeSe $_{1-x} \mathrm{~S}_{x}$ single crystals were obtained up to $19 \%$ sulfur content using the innovative "ampoule in ampoule" technique of crystal growth under permanent gradient of temperature in the mixture of molten metal chlorides. This method allows obtaining large size high quality single crystals with a wide range of S-substitution levels. Non-stoichiometry does not depend strongly on the sulfur content and is the same as the non-stoichiometry in tetragonal FeSe. Scanning tunneling microscopy reveals the presence of dumbbell defects associated with $\mathrm{Fe}$ vacancies. In the S-substituted crystals, depressions are observed at the chalcogen lattice and are associated with sulphur atoms that have smaller radii than selenium. Scanning tunneling spectroscopy evidences the presence of two different superconducting gaps at both hole and electron pockets of the Fermi surface, which do not change significantly at low S-substitution (4\%). As a function of sulfur content, $T_{\mathrm{C}}$ follows the conventional dome-shaped curve while $T_{\mathrm{N}}$ decreases with $x$. The overall appearance of the $T-x$ phase diagram of $\mathrm{FeSe}_{1-x} \mathrm{~S}_{x}$ suggests importance of nematic fluctuations for the formation of the superconducting state in these compounds.

\section{Conflicts of interest}

The authors contributed equally and declare no competing interests.

\section{Acknowledgements}

This work was supported by the Ministry of Education and Science of the Russian Federation in the framework of the Increase Competitiveness Program of NUST "MISiS" grant K22017-084, by Act 211 of the Government of Russian Federation, Contracts No. 02.A03.21.0004, 02.A03.21.0006, 02.A03.21.0011 and by the Russian Government Program of Competitive Growth of Kazan Federal University. The work in Germany was supported by the program MO30214/2. The work at Temple
University, where low temperature scanning tunneling measurements were performed, was supported by the U.S. Department of Energy under Grant No. DE-SC0004556.

\section{References}

1 T. M. McQueen, A. J. Williams, P. W. Stephens, J. Tao, Y. Zhu, V. Ksenofontov, F. Casper, C. Felser and R. J. Cava, Phys. Rev. Lett., 2009, 103, 057002.

2 Y. Mizuguchi and Y. Takano, J. Phys. Soc. Jpn., 2010, 79, 102001.

3 F.-C. Hsu, J.-Y. Luo, K.-W. Yeh, T.-K. Chen, T.-W. Huang, P. M. Wu, Y.-C. Lee, Y.-L. Huang, Y.-Y. Chu, D.-C. Yan and M.-K. Wu, Proc. Natl. Acad. Sci. U. S. A., 2008, 105, 14262.

4 Y. Mizuguchi, F. Tomioka, S. Tsuda, T. Yamaguchi and Y. Takano, Appl. Phys. Lett., 2008, 93, 152505.

5 B. Lei, J. H. Cui, Z. J. Xiang, N. Z. Wang, C. Shang, N. Z. Wang, G. J. Ye, X. G. Luo, T. Wu, Z. Sun and X. H. Chen, Phys. Rev. Lett., 2016, 116, 077002.

6 M. Abdel-Hafiez, Y.-Y. Zhang, Z.-Y. Cao, C.-G. Duan, G. Karapetrov, V. M. Pudalov, V. A. Vlasenko, A. V. Sadakov, D. A. Knyazev, T. A. Romanova, D. A. Chareev, O. S. Volkova, A. N. Vasiliev and X.-J. Chen, Phys. Rev. B: Condens. Matter Mater. Phys., 2015, 91, 165109.

7 M. D. Watson, T. K. Kim, A. A. Haghighirad, S. F. Blake, N. R. Davies, M. Hoesch, T. Wolf and A. I. Coldea, Phys. Rev. B: Condens. Matter Mater. Phys., 2015, 92, 121108.

8 P. Reiss, M. D. Watson, T. K. Kim, A. A. Haghighirad, D. N. Woodruff, M. Bruma, S. J. Clarke and A. I. Coldea, Phys. Rev. $B, 2017,96,121103$.

9 H. C. Hu, X. H. Niu, D. F. Hu, J. Jiang, Q. Yao, Q. Y. Chen, Q. Song, M. Abdel-Hafiez, D. A. Chareev, A. N. Vasiliev, Q. S. Wang, H. L. Wo, J. Zhao, R. Peng and D. L. Feng, Phys. Rev. Lett., 2016, 117, 157003.

10 H. Okamoto, J. Phase Equilib., 1991, 12, 383.

11 F. Gronvold, Acta Chem. Scand., 1968, 22, 1219.

12 L. A. Taylor and L. W. Finger, Geophys. Lab. Ann. Rept., Carnegie Institute of Washington, 1970, vol. 69, p. 318.

13 H. Zoka, L. A. Taylor and S. Takeno, J. Sci. Hiroshima Univ., Ser. C, 1973, 7, 37.

14 D. J. Vaughan and J. R. Craig, Mineral Chemistry of Metal Sulfides, Cambridge Univ. Press, Cambridge, 1978.

15 X. Lai, H. Zhang, Y. Wang, X. Wang, X. Zhang, J. Lin and F. Huang, J. Am. Chem. Soc., 2015, 137, 10148.

16 S. G. Parker and J. E. Pinnell, J. Cryst. Growth, 1968, 3, 490.

17 J.-Q. Yan, B. C. Sales, M. A. Susner and M. A. McGuire, Phys. Rev. Mater., 2017, 1, 023402.

18 R. Hu, H. Lei, M. Abeykoon, E. S. Bozin, S. J. L. Billinge, J. B. Warren, T. Sigrist and C. Petrovic, Phys. Rev. B: Condens. Matter Mater. Phys., 2011, 83, 224502.

19 D. Chareev, E. Osadchii, T. Kuzmicheva, J.-Y. Lin, S. Kuzmichev, O. Volkova and A. Vasiliev, CrystEngComm, 2013, 15, 1989.

20 D. A. Chareev, O. S. Volkova, N. V. Geringer, A. V. Koshelev, A. N. Nekrasov, V. O. Osadchii, E. G. Osadchii and O. N. Filimonova, Crystallogr. Rep., 2016, 61, 682. 
21 S. Margadonna, Y. Takabayashi, M. T. McDonald, K. Kasperkiewics, Y. Mizuguchi, Y. Takano, A. N. Fitch, E. Suard and K. Prassides, Chem. Commun., 2008, 5607.

22 Y. Mizuguchi, F. Tomioka, S. Tsuda, T. Yamaguchi and Y. Takano, J. Phys. Soc. Jpn., 2009, 78, 074712.

23 D. Huang, T. A. Webb, C.-L. Song, C.-Z. Chang, J. S. Moodera, E. Kaxiras and J. E. Hoffman, Nano Lett., 2016, 16, 4224.

24 S. Kasahara, T. Watashige, T. Hanaguri, Y. Kohsaka, T. Yamashita, Y. Shimoyama, Y. Mizukami, R. Endo, H. Ikeda, K. Aoyama, T. Terashima, S. Uji, T. Wolf, H. von Lohneisen, T. Shibauchi and Y. Matsuda, Proc. Natl. Acad. Sci. U. S. A., 2014, 111, 16309.

25 T. Watashige, Y. Tsutsumi, T. Hanaguri, Y. Kohsaka, S. Kasahara, A. Furusaki, M. Sigrist, C. Meingast, T. Wolf, H. von Lohneysen, T. Shibauchi and Y. Matsuda, Phys. Rev. X, 2015, 5, 031022.

26 T. M. McQueen, A. J. Williams, P. W. Stephens, J. Tao, Y. Zhu, V. Ksenofontov, F. Casper, C. Felser and R. J. Cava, Phys. Rev. Lett., 2009, 103, 057002.

27 C.-L. Song, Y.-L. Wang, P. Cheng, Y.-P. Jiang, W. Li, T. Zhang, Z. Li, K. He, L. Wang, J.-F. Jia, H.-H. Hung, C. Wu, X.-C. Ma, X. Chen and Q.-K. Xue, Science, 2011, 332, 1410 .
28 C.-L. Song, Y.-L. Wang, Y.-P. Jiang, L. Wang, K. He, X. Chen, J. E. Hoffman, X.-C. Ma and Q.-K. Xue, Phys. Rev. Lett., 2012, 109, 137004.

29 P. O. Sprau, A. Kostin, A. Kreisel, A. E. Bohmer, V. Taufour, P. C. Canfield, S. Mukherjee, P. J. Hirschfeld, B. M. Andersen and J. C. S. Davis, Science, 2017, 357, 75.

30 S. A. Moore, J. L. Curtis, C. Di Giorgio, E. Lechner, M. AbdelHafiez, O. S. Volkova, A. N. Vasiliev, D. A. Chareev, G. Karapetrov and M. Iavarone, Phys. Rev. B: Condens. Matter Mater. Phys., 2015, 92, 235113.

31 M. Abdel-Hafiez, J. Ge, A. N. Vasiliev, D. A. Chareev, J. Van de Vondel, V. V. Moshchalkov and A. V. Silhanek, Phys. Rev. B: Condens. Matter Mater. Phys., 2013, 88, 174512.

32 M. Abdel-Hafiez, Y. J. Pu, J. Brisbois, R. Peng, D. L. Feng, D. A. Chareev, A. V. Silhanek, C. Krellner, A. N. Vasiliev and X.-J. Chen, Phys. Rev. B, 2016, 93, 224508.

33 S. Hosoi, K. Matsuura, K. Ishida, H. Wang, Y. Mizukami, T. Watashige, S. Kasahara, Y. Masuda and T. Shibauchi, Proc. Natl. Acad. Sci. U. S. A., 2016, 113, 8139.

34 K. Matsuura, Y. Mizukami, Y. Arai, Y. Sugimura, N. Maejima, A. Machda, T. Watanuki, T. Fukuda, T. Yajima, Z. Hiroi, K. Y. Yip, Y. C. Chan, Q. Niu, S. Hosoi, K. Ishida, K. Mukasa, S. Kasahara, J. G. Cheng, S. K. Goh, Y. Matsuda, Y. Uwatoko and T. Shibauchi, Nat. Commun., 2017, 8, 1143. 